\title{
Computer programs - new considerations in teaching and learning mathematics science
}

\author{
Qamil Kllogjeri ${ }^{1,}$, Pellumb Kllogjeri ${ }^{1,2}$ \\ ${ }^{1}$ University of Gjovik, MSc Student of Information Security, Gjovik, Norway \\ ${ }^{2}$ University "Aleksander Xhuvani ", Department of Mathematics and Informatics, Elbasan, Albania
}

Email address:

q.kllogjeri@gmail.com (Qamil Kllogjeri),pkallogjeri@gmail.com (Pellumb Kllogjeri)

\section{To cite this article:}

Qamil Kllogjeri, Pellumb Kllogjeri. Computer Programs - New Considerations in Teaching and Learning Mathematics Science. American Journal of Software Engineering and Applications. Special Issue: Growing GeoGebra Environment and Use. Vol. 4, No. 2-1, 2015 , pp. 7-13. doi: $10.11648 /$ j.ajsea.s.2015040201.12

\begin{abstract}
There are many applications of computer informatics like in computations, plotting graphics to use them in math papers and to study the properties of the functions, in solving and discussing problems of mathematics or physics, in economics, in social topics and so on. This paper presents the topic of how much the computer programs (while we are studying something or making some trials by manipulating) help the teacher in finding answer for different mathematical problems or for the formulation of mathematical statements or facts (in other fields of science, as well). We are presenting here several examples in order that teachers and students have them into consideration while using computer programs to teach and learn. It is important that the teachers and the students try themselves again these examples and others by manipulating with computer programs, making trials and keeping notes in order to find out that there are limitations in the computer programs. The computer program used is Geogebra.
\end{abstract}

Keywords: GeoGebra, Virtual Tools, Discrete Medium of Computer Programs, Limitations of Computer Programs

\section{Introduction}

The computers age has fundamentally changed many things in our culture. The ways we learn mathematics and do mathematics and, the kinds of problems we can consider and solve have changed. Our creative visions and our sense of what is possible have been altered. The computers are as discrete machines, capable of dealing only with finite information. Discrete mathematics deals with many real-world applications and it has very close ties with computer science. The range of applications of both discrete mathematics and computer science is growing so rapidly that becomes so difficult to decide what program or what algorithm is better.

Applications of discrete mathematics include project communication networks, systems analysis, management, social decision making, population growth, and finance.

The wide range of the applications oblige the teachers to provide students the knowledge and skills of discrete mathematics to prepare them for life-work by positively responding to these fast changes in sciences and in culture.

In the teaching and learning process, also in applications, are treated and used continuous mathematics and discrete mathematics. Continuous mathematics is the mathematics based on the continuous number line or the real numbers. The simplest meaning is: for given any two numbers we can always find another number between them, furthermore we can find an infinite set of numbers between them. Geometrically, a function is continuous if its geometric representation is a perfectly smooth curve, without any gaps.

Discrete mathematics is the mathematics based on distinct values. Geometrically speaking, given any two numbers (points), there aren't an infinite number of numbers (points) between them. So, in the teaching and learning process the teachers have to take into consideration that the computers are as discrete machines that deal only with finite information. We are not sure that a computer program written to compute an expression $\mathrm{S}$ is correct. For instance, let be the expression: $\mathrm{S}=$ $(\mathrm{a}+\mathrm{b})^{\mathrm{n}}$. There are infinitely many possible input values (that is, values of $a, b$ and $n$ ). Who and what can be able to test the correctness of the program for infinitely many cases?!

There is another approach related to the problem of correctness of computer programs. This is the well-known "Halting Problem". This problem asks whether or not a given computer program stops on a given input after a finite amount 
of time. This problem is known to be unsolvable by computers, as well. No one can write a computer program to answer the above question (John Tabak, 59).

Discrete mathematics is the foundation for the formal approaches. It discusses languages used in mathematical reasoning, basic concepts, and their properties and relationships among them.

GeoGebra is a computer software, it is a dynamic mathematics software that joins geometry, algebra and calculus (Josef Böhm-2008). It is an interactive geometry system with which we can make different constructions using geometry virtual tools offered by this software or, by directly entering into the input field equations, functions and points coordinates that are their algebraic representatives. We can also change them dynamically. Characteristic of GeoGebra are two views of the object in study: the algebraic expression of the geometric object in the algebra window and the object in the geometry window, and the two windows can be seen simultaneously (Markus Hohenwarter, Judith Hohenwarter, 2008). The graphical representation of all objects is displayed in the graphics window (geometry window), while their algebraic representations are shown in algebra window. Any change done in the object is reflected with changes in its algebraic representation and vice versa.

The benefits of using computer programs in solving different problems are well-known. In the following explorations we are drawing the attention of the teachers, including the program users as well, to keep in mind that there are limitations as to the scale the computer programs are used in micro-world or macro-world. The explorations are done using GeoGebra software.

\section{Limitations of Computer Programs in Exploring Mathematical Issues}

\subsection{The Problem of Intersection of Two Circles}

The problem of the intersection of two circles regarding the determination of the intersection points can be easily studied by using a computer program, and a very good software is GeoGebra with which we can get geometric and algebraic answer (Lu Yu-Wen Allison, pp.2). Let see some examples in referee to this problem. In the obvious cases the demonstration of the fact that the circles are intersected or not is done in a very easy way. It is understandable by the students and there is no question raised.

For instance, the intersection of the circles with equations

$$
x^{2}+y^{2}=4 \text { and }(x-1)^{2}+y^{2}=4
$$

is seen by the geometric construction.

If we take the circles

$$
x^{2}+y^{2}=4 \text { and }\left(x-4+10^{-6}\right)^{2}+y^{2}=4
$$

where the second equation can be written as

$$
\left(x-\frac{399,999}{100,000}\right)^{2}+y^{2}=4
$$

the geometric view shows that they are tangent, but by using the intersection tool of GeoGebra (Intersect Two Objects) to find the intersection points of the two circles we get the right answer of two intersection points.

Taking other examples by choosing the circle center close to point $(4,0)$ and on its left, with the same radius $(r=2)$, the problem of detecting the intersection points becomes more difficult. Taking the circle

$$
c:\left(x-4+10^{-10}\right)^{2}+y^{2}=4
$$

it is noticed that it is registered as $(x-4)^{2}+y^{2}=4$ in the algebra window.

Using Intersect Two Objects tool to find the intersection points of the two circles we get the answer of two intersection points: $\mathrm{D}(2,0)$ and $\mathrm{E}(2,-0)$. This is an approximate numerical answer for their coordinates.

GeoGebra offers tools to see more details in the graphical representation of the object.

So, applying the Zoom in tool (by which the picture is magnified), as much as the program allows, we detect the two points $\mathrm{D}$ and $\mathrm{E}$ close to point $(2,0)$, and there is only one point on the circle: $x^{2}+y^{2}=4$. In fact, there are two different points on the two circles.

This fact shows that the program cannot give the right answer about the intersection of the two circles when their central distance is very close to number 4 and less than it.

Take the circle $c$ with equation,

$$
\left(x-4+10^{-15}\right)^{2}+y^{2}=4
$$

which is again registered in the Algebra window as,

$$
(x-4)^{2}+y^{2}=4
$$

The central distance between the circles

$$
\left(x-4+10^{-15}\right)^{2}+y^{2}=4
$$

and $x^{2}+y^{2}=4$ is much, much closer to number 4 and less than it.

Applying the Intersect Two Objects tool, to find the intersection points of the two circles, we get the answer of two intersection points in the Algebra window, appeared as $\mathrm{D}(2,0)$ and $\mathrm{E}(2,0)$. That means: one point!!! Then, applying Zoom in tool, as much as the program allows, we see no different intersection points. This is a stronger confirmation that the computer program is limited, it cannot give answer for every problem we present to it for solution. They are of limited use in micro-world.

\subsection{The Problem of Intersection and Parallelism}

Let see now the problem of parallelism of the lines using GeoGebra software. Entering into the Input field the following line equations

$$
y=2 \cdot x-3 \text { and } y=\frac{1}{2} \cdot x+1,
$$

it is easily demonstrated by the program that they are intersected, that means they are not parallel. We choose other 
equations that their angular coefficients differ very little from one another. Let us take the example of the lines with equations:

$$
a: y=10^{-8} \cdot x+5 ; b: y=2 \cdot 10^{-8} \cdot x-5
$$

The way to check if the lines are parallel or not, by using GeoGebra program, is calculating the distance between them. If the lines are intersected the distance between them is zero, and if the lines are parallel the distance between them is a positive number. Using the Distance tool in this case we get the right answer: the distance between them is zero (because they intersect one another). We take another example, in which the angular coefficients of the lines differ very, very little from one another. We enter into the Input field the equations:

$$
c: y=10^{-9} \cdot x+5 ; d: y=2 \cdot 10^{-9} \cdot x-5
$$

They are simply registered as $y=5$ and $y=-5$ in Algebra window.

The "distance" command shows that the distance between them is 10 !!!, meaning they are parallel, contrary to the fact that they are not parallel. Applying Zoom out tool (because this command moves much faster the pane to the "Far East" or "Far West" of the coordinative plane), as far as the program allows, and later applying Zoom in tool to magnify the area at these extremes in order to see the parts of the lines, they look parallel !!!?.

Entering the equations,

$$
f: y=10^{-10} \cdot x+5 ; g: y=2 \cdot 10^{-10} \cdot x-5
$$

they are also registered as $\mathrm{y}=5$ and $\mathrm{y}=-5$, and the distance between them is 10 , meaning they are parallel !!! (in fact, the two lines intersect). We can bring many other examples.

The same conclusion can be drawn regarding the parallelism axiom of the lines. From a point A, outside a line $d$ passes through only one line d' parallel to $d$. But, this fact cannot be demonstrated by GeoGebra software. We can construct a great number of lines passing through the point $\mathrm{A}$ for which their angular coefficients differ from one another and from d' by a very small amount. All they are different, but Geometry window shows that they take the position of the line d'!!!

Here again, we are facing a problem that the computer program cannot demonstrate the property of intersection or parallelism for the lines. GeoGebra does not help us to get right answers in every case of macro-world as well.

\subsection{Can the Program Demonstrate that any Two Lines Can be Perpendicular or not?}

Consider the lines with equations: $y=2 x$ and $y=-0.5 x$. Entering these equations in the Input field are got the two lines in Geometry window, and measuring the angle between them by using the angle tool is shown that it is $90^{\circ}$. This is a true fact because the production of their angular coefficients is -1 as well. We take another equation, $\mathrm{y}=-0.4999 \mathrm{x}$, enter it in the Input field and measure the angle between the line of this equation and the line $\mathrm{d}(\mathrm{y}=2 \mathrm{x})$. The answer is $90^{\circ}$, meaning that they are perpendicular as well. In fact, they are not because the production of their angular coefficients is not -1 . We can take a great number of lines with equations: $y=-0.49999 x, y=-0.499999 x, \ldots$, and so on. Constructing their respective graphs by entering their equations in the Input field we notice that all the respective lines take one position: there is no difference between them. Measuring the angle between them (represented by a line alone) and the line $\mathrm{d}$ we get the same answer by the program: the measured angle is $90^{\circ}$ Actually, the lines must take different positions. They have only one point in common: the intersection point which is the origin of coordinative system. Taking four different equations, constructing their respective lines, and using Zoom In tool, as many times as the program allows, to see if their positions are different we don't notice such thing. There is only one line passing through the or*igin. Thinking that the region we are looking at is very bounded and very close to the origin we use the Move Graphic View tool to move the geometry window on the left or on the right side and along the line. Moving on the left, and "far west", and checking the positions, we observe that all the lines $\mathrm{y}=\mathrm{kx}$, for which the angular coefficient satisfies the condition:

$$
\begin{gathered}
|k-(-0.5)|=|k+0.5| \leq 10^{-4} \Rightarrow \\
-0.5001 \leq k \leq-0.4999,
\end{gathered}
$$

take the same position. The lines coincide. Maybe there are other lines as well that are present here, so anyone can try by taking $k$ : $-0.501<k<-0.499$. Definitely, the computer program cannot demonstrate that any two lines are perpendicular or not.

\subsection{Can the Program Demonstrate that Any Two Circles Can be Tangent}

Consider the circles: $x^{2}+y^{2}=4$ and $(x-4)^{2}+y^{2}=$ 4. They are tangent, but try using some computer program what they show. We have used GeoGebra. They look tangent in the normal view of Geometry window. Then, we use the Zoom In tool moving towards micro-world to see if it is true that they have one point in common. Without making such effort we know that the point is dimensionless, hence nobody has to expect that will find some extraordinary thing. Using Zoom In tool, as many times as the program allows, we see within the Geometry view only a common line of the two circles. We try to move deeper and deeper towards the micro-world, expecting to see a split of the curved parts of the two circles, but it doesn't happen. Furthermore, we come to a moment when the line disappears from our sight. Then, we move back, using Zoom Out tool, until the line appears again and try another movement. Using the Move Graphic View tool, we displace the view down in order to move up and "far-north". We continue moving up, hoping to see the split, until the ordinates of the points of the common line reach the value 2 . We have a big part of the common points of the two circles that is checked using Geogebra software (their ordinates are between 0 and 2), and no split!!! It is enough to say that the program cannot demonstrate that the two circles 
have in common one point alone.

\subsection{Can a Program Show that ANY Equation of Type $\frac{x^{2}}{a^{2}}+\frac{y^{2}}{b^{2}}=1, a^{2} \neq b^{2}$ is Represented by an Ellipse?}

Consider the equation $\frac{x^{2}}{2.0009^{2}}+\frac{y^{2}}{2^{2}}=1$.

Entering this equation in the Input field we get in the Geometry window a circle. Sure, this is depended on our sight ability. Therefore we rely on the program: what does it say? Constructing the intersection points of the geometrical representation of the equation with the coordinative axes we get the points: $(2,0),(-2,0),(0,2)$, and $(0,-2)$ which means that the two ellipse axes (major and minor) are equal. The program cannot make the difference between the given ellipse and the circle with equation $x^{2}+y^{2}=4$. In general, the program does make a difference between this circle and the ellipses with equations $\frac{x^{2}}{a^{2}}+\frac{y^{2}}{2^{2}}=1$ if $a^{2}>2.0009^{2}$ or $a^{2}<$ $1.9991^{2}$. For instance, if $a^{2}=2.009^{2}$ then the intersection points of the ellipse with the coordinative axes are: $(2.1,0)$, $(-2.1,0),(0,2)$ and $(0,-2)$.

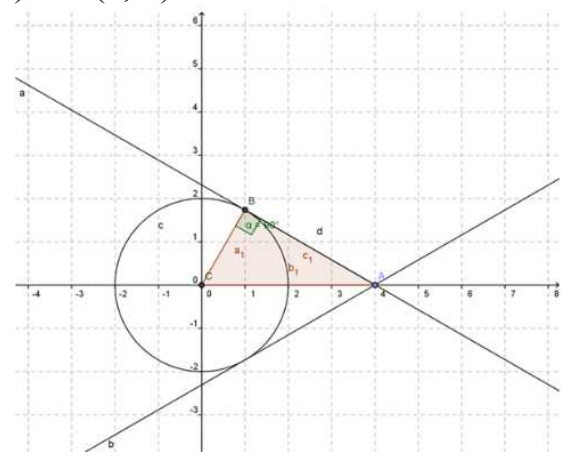

Fig 1. Right angled triangle.

\subsection{Can be Proved or Disapproved by Using a Program that a Triangle is a Right Angled One?}

We know that in a right angled triangle the longest side of the triangle is called "hypotenuse". So, the formal definition (based on Pythagoras' Theorem) is: In a right angled triangle, the square of the hypotenuse is equal to the sum of the squares of the other two sides. Using GeoGebra we construct the circle of radius 2 (look at Figure 1) and from point $A=(4,0)$ are constructed its tangents. Consider the tangent $\mathrm{AB}$ and the triangle ABC. Using the Angle tool we measure the angle ABC. In Figure 1 is shown that the size of the angle is: $\alpha=90^{\circ}$. This fact is based on what we see and what the program shows The only precise way of proving that a triangle is right angled is to check if the Pythagorean Theorem is true. In our case, using GeoGebra tools, we are given the lengths of hypotenuse $\mathrm{AC}=\mathrm{b}_{1}=4$, of the sides $\mathrm{AB}=\mathrm{c}_{1}=3.46$ and $\mathrm{BC}=\mathrm{a}_{1}=2$. We check weather $b_{1}^{2}=a_{1}{ }^{2}+c_{1}{ }^{2}$ is valid, but $4^{2} \neq 2^{2}+3.46^{2}$ !!! This result depends on the size of the error done during the calculation of the length of the side $c_{1}$. Nevertheless, whatever small be the error, the program cannot demonstrate that the given triangle has a right angle!! We could take another example in which are given the lengths of the three sides of a triangle, and using the angle tool by measuring the biggest angle we could find out that it is $90^{\circ}$ !! This is not the right way to prove that a triangle is right angled.

\subsection{The Optical Property of the Ellipse}

Suppose a line $a$ is tangent to an ellipse at a point T. Then, the angles between the line $a$ and the two foci rays (TF1 and TF2), drawn from point $T$, are equal.

We can demonstrate this property using GeoGebra software (look at Figure 2) for the ellipse

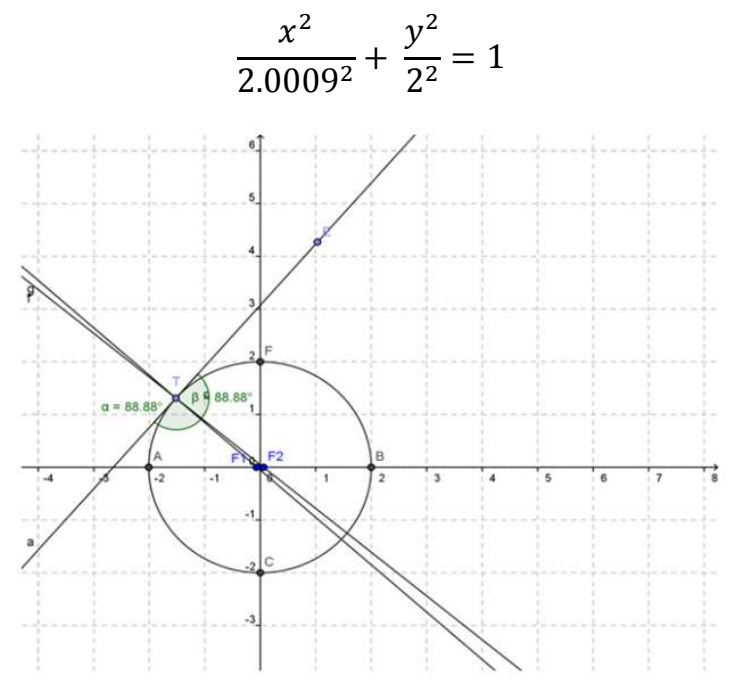

Fig 2. The property of foci rays.

This is also true for other ellipses for which the first semi-axis satisfies the condition: $a^{2}>2.0009^{2}$. But if we take another example like

$$
\frac{x^{2}}{2.00009^{2}}+\frac{y^{2}}{2^{2}}=1
$$

or in general, satisfying the condition $a^{2}<2.0009^{2}$, then the foci are very close to the coordinative origin. The program does not help to demonstrate the above property of the foci rays because, in such cases, the foci rays are represented by a line alone: they are placed one on the other. In reality the situation is not so, they are different but the program shows one line.

\subsection{The Difference between the Areas of a Circle and the Regular Polygon Inscribed in it (Case: the Side Length Decreases)}

Consider the circle with radius $r=6$. Before constructing the inscribed regular polygon of the circle we insert in the Geometry window a slider, named $n$, which has the function of changing the number of the sides of the regular polygon in order to observe what happens with the two areas when the number $\mathrm{n}$ of the sides is increased. To construct the regular $n$-gon, inscribed in the circle, we construct a vertex with coordinates:

$$
M=\left(6 \cos \frac{360^{\circ}}{n}, 6 \sin \frac{360^{\circ}}{n}\right)
$$


which falls on the circle. Construct the intersection point A of the circle with the $x$-axis on the positive side. Then we use the Regular Polygon tool referring the segment AM as one of its sides. Using the slider $n$ we increase the number of the sides of the regular polygon to observe the difference between the area of the circle and the area of the regular $n$-gon. In this case the side length decreases. The first observations for the differences, where the number of sides is considerably great, are shown in the table below (Table 1): for greater values of $n$ the difference remains zero. We use Zoom In tool for the regular 2000-gon to look closer at the relation between one side and the respective arc of the circle. The observation shows that they have different positions, meaning that there is a part of the circle which is outside the polygon. This is true for the other sides of the regular polygon and their respective arcs.

Table 1. The difference between the area of the circle and the area of the regular $n$-gon inscribed in it

\begin{tabular}{lllllll}
\hline$n$ & 50 & 100 & 300 & 500 & 1000 & 1500 \\
Differences & 0.3 & 0.08 & 0.01 & 0.01 & 0 & 0 \\
\hline
\end{tabular}

So, there are 2000 small pieces of the circle that are outside the polygon, hence the difference between the circle area and the regular polygon, inscribed in, is not zero (it is very, very small but not zero). We get the difference zero because there are limitations in the program with respect to calculations. However sophisticated the program be it cannot give the answer for any problem in micro-world or in macro-world.

\subsection{The Difference between the Areas of a Circle and the Regular Polygon Circumscribed around it (Case: the Side Length is Constant)}

Consider a circle with radius $R$ and a regular $n$-gon circumscribed around the circle (Fig. 3 ). Let the side of the regular polygon be of a fixed length denoted by $a$. We observe the difference between the area of the circumscribed regular $n$-gon and the area of the circle which is tangent with all the sides of the polygon. The observation is done using Geogebra software and for the case $a=4$. The number of the sides is changed by using the slider $n$, as in the previous example. We have constructed the two objects in such a way that, when the number $n$ of the sides is changed, the two objects change simultaneously and their relation is not affected. That is, whatever be the value of $n$, the regular $n$-gon is circumscribed around the circle (both they represent one object). The results of the observation are presented in the following table (Table 2):

Table 2. The difference between the area of the circle and the area of the regular $n$-gon circumcised around it

\begin{tabular}{lllllll}
\hline$n$ & 18 & 120 & 500 & 1000 & 2000 & 3000 \\
Differences & 4.17 & 4.2 & 4.2 & 4.2 & 4.2 & 4.2 \\
\hline
\end{tabular}

The observation shows that the different values of the difference stand for small values of $n$. As can be seen from the table, for great values of $n$ there is no change.

The program does not help in observing this phenomenon. In other words, it cannot give the answer about what the series of differences is: decreasing or increasing? Analytical methods give the right answer. There is no place here to show that the differences of the areas represent a decreasing series. We just calculate the limit of the ratio of the area of the regular $n$-gon, circumscribed around the circle, with the area of the respective circle where, $R=\mathrm{GH}, a=\mathrm{AB}$ ( $a$ is the length of any side), $n$ is the number

of sides and $\pi$ is approximately $3.142, \mathrm{G}$ is the center of the circle.

$$
\begin{gathered}
\operatorname{area}(\triangle O A B)=\frac{a}{2} \cdot R=\frac{a^{2}}{4 \cdot \tan \frac{\pi}{n}} \\
\operatorname{area}\left(\operatorname{sector} \frac{2 \pi}{n}\right)=\frac{\pi \cdot R^{2}}{n}=\frac{\pi}{n} \cdot \frac{a^{2}}{4 \cdot\left(\tan \frac{\pi}{n}\right)^{2}} \\
\lim _{n \rightarrow \infty} \frac{\operatorname{area}(\Delta)}{\operatorname{area}(\operatorname{sector})}=\lim _{n \rightarrow \infty} \frac{\frac{a^{2}}{4 \cdot \tan \frac{\pi}{n}}}{\frac{\pi}{n} \cdot \frac{a^{2}}{4 \cdot\left(\tan \frac{\pi}{n}\right)^{2}}}=\lim _{n \rightarrow \infty} \frac{n}{\pi} \tan \frac{\pi}{n} \\
=1
\end{gathered}
$$

The result of the limit, which is 1 , of the ratio (when the number of the sides is infinitely increased) shows that the areas of the circle and of the regular $n$-gon, circumscribed around it, tend to equalize with one another. Hence, it is understood that the series of the above observed differences is a decreasing one.

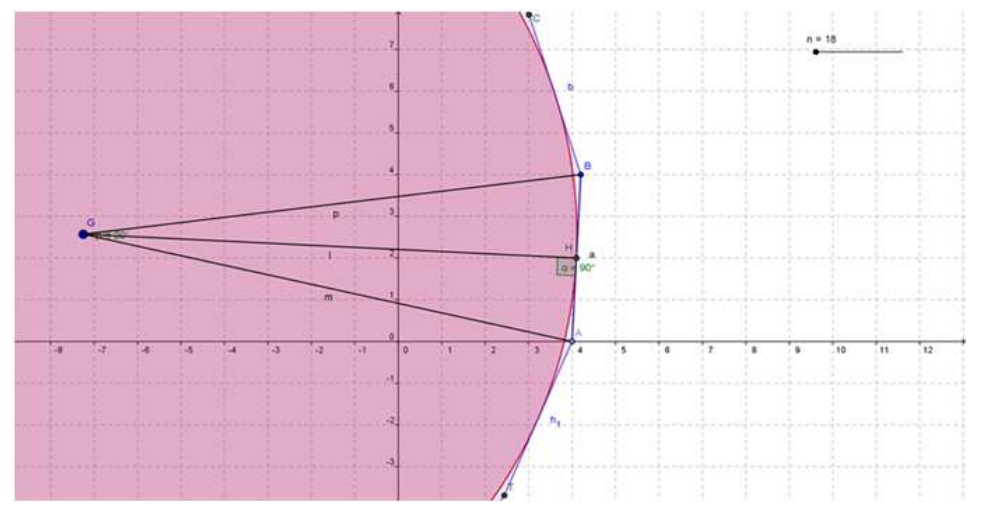

Fig 3. The circle and the regular n-gon circumscribed around it. 


\subsection{The area Problem of Circles in the Micro-World}

Another example, showing that the computer program is limited, is the circle of a very small radius. Entering in the Input field equations of the form $x^{2}+y^{2}=r^{2}$ it is possible to take the constructions for the cases: $r^{2}=10^{-2}, . . r^{2}=10^{-4}, \ldots$. until.. $r^{2}=10^{-8}$. Applying Zoom In tool we can clearly see the circles for which $r^{2}=10^{-4} .$. and $. . r^{2}=10^{-5}$. In this micro-world they look big and very far from one another, but if we calculate their areas, they are zero.

\section{The Medium in which the Program Operates is Really Discrete}

The set of rational numbers has the property: between any two rational numbers there are also infinitely many rational and irrational numbers. This is called: property of being "dense", because it means that we can find rational numbers in even the smallest gaps on the number line. Always can be answered the question: if are given two different rational numbers, is it possible to find another rational number that sits between them?

Let see if this property is supported by a computer program. We take the rational numbers $1 / 3$ and $2 / 3$ and explore how many different numbers between them can be found using GeoGebra software. Sure, can be found a lot, but to what scale can the program help? The best way to explore this property is to take segments with ends representing the rational numbers we are seeking. The dense property of the rational numbers can be formulated in a geometrical way: for any segment with ends in different rational numbers can be found segments (also infinitely many) with ends in rational numbers that are lied within the first segment. So, we start with the segment with ends at the points: $(1 / 3,0)$ and $(2 / 3,0)$ which is clearly visible in the Geometry window lied on the $\mathrm{x}$-axis. Then, we take the segment with ends at the points: $(7 / 15,0)$ and $(1 / 2,0)$. It is visible that it is within the first segment, but too short. We color them with different colors in order to make the distinction. Then, we take the segment with ends at the points: $(145 / 300,0)$ and $(148 / 300,0)$. The segment is not visible in the normal view therefore we use the Zoom In tool to bring it to our sight. It becomes visible and lies within the second segment.

Continuing further by taking the segment with ends at the points $(1456 / 3000,0)$ and $(1475 / 3000,0)$ and using Zoom In tool we can detect that this segment lies within the third segment. For further exploration we take the segment with ends at the points: $(146 / 300,0)$ and $(147 / 300,0)$. Using Zoom In tool we can detect that this segment lies within the fourth segment. Trying to go deeper by selecting segments with ends within the last one is very difficult. There are still infinitely many of such segments but the program doesn't help to detect them. For instance, the segment with ends at the points: $(1456 / 3000,0)$ and $(1460 / 3000,0)$ cannot be shown by the program. Using Zoom In tool we cannot detect this segment, it is missing. The result of this exploration is that between the points generated by the program there are vast gaps. Surely, the points, generated by the program, have a real size that is extremely big compared to very tiny elements of the micro-world. The mathematical meaning of the point is as a dimensionless object. This experiment fully confirms the results and conclusions of the former examples that the medium in which the program operates is discrete

\section{Last Thoughts}

We brought many examples or facts that show that the computer programs help for exploring mathematical issues down or up to a level, and moving further is impossible. Using the popular language we have reached a point where the saw has faced a nail or stuck in it.

It happens this way because many of our basic intuitions about numbers and the things that we can do with them are actually deeply connected with the limits of computation. There are also people of such arguments who try to invoke ideas about computation in misleading ways. In the history of mathematics are known the efforts done in the early 20th century to create a perfect complete mathematics, which meant a new formalization of mathematics in terms of a logic in which every true statement was probably true, every false statement was provably false, and for any given statement could be followed a completely mechanical process to find out whether it was true or false (Bruner, 21-32).

Gödel wrestled with his incompleteness theorems as well. Later, Alan Turing worked on defining the limit of what could be done by a purely mechanical computation process. What Turing showed about computability was really the same concept as what Gödel showed about logic with incompleteness: there are limits of mechanical/axiomatic processes (Michel Detlefsen, pp.26). By remembering this short history we want to emphasize that Turing did prove that there are things that cannot be computed. He did it by defining an easy to understand problem which cannot be solved by any mechanical device, and by showing how common non-computable things are.

The first of Turing's proofs is known as the Halting problem. The simplest version of the Halting problem is the following:

Suppose that some program $\mathrm{P}$ can be run on a computing machine M. Can someone write a program $\mathrm{H}$ which can determine whether the running $\mathrm{P}$ will eventually stop? In this case we have a computing machine which computes some function on its input. The halting problem (John Tabak, 59) is in fact the question: given a machine $M$, is there such a program $\mathrm{H}$ that at the end will result with the answer "YES" (return YES) if and only if M would halt, and will return "NO" if and only if $\mathrm{M}$ would not halt. Such a program $\mathrm{H}$ is called a halting oracle and the question is raised differently: is it possible to write a halting oracle?

The answer is no because no such program can possibly exist. It is very easy to write a program for which $\mathrm{H}$ is guaranteed to get the wrong answer. On the other side there are 
lots of things that can't be computed. Actually, most functions are not computable! Is the same thing with the numbers, most of which are irrational numbers. So, most of the functions are not computable. Let consider just the functions on the natural numbers: $\{\mathrm{f} \mid \mathrm{f}: \mathrm{N} \rightarrow \mathrm{N}\}$. It is an infinite set of such functions which is larger than the infinite set of natural numbers. Turning back to a computing machine, we can reflect each one of its programs to a natural number. Consequently, the computing machine can only have as many programs as there are natural numbers. So, there are values in that infinite set of functions that have no program that can compute them.

We have brought here facts that bring to light the limitations of the computer programs. Here it is important to turn that truth into understanding. This is linked with the interpretation of the data. Do we understand how the program runs or works? How precise and how well understood are the outputs of the program? These are results of explorations that are carefully done. The explorations or the experiments can, in fact, be wrong but the facts aren't wrong. It is possible to make mistakes in our interpretation of the facts. Good experimentalists usually do things right, but we all make mistakes. There are cases when we do everything right which is associated with something going on that we are not aware of it. We cannot know everything that is linked with our experiment or exploration. Once we have our experimental results, we set to the task of trying to understand them, trying to put them in context and turn them into something that we might call an understanding of some part of nature (Hulshof, pp. 27; Reimann P, pp.34). The ultimate goal is to develop a fruitful and working theory.

A theory is a framework for understanding how does the nature work, how a process works and it serves as means of predicting the results of experiments and observations (Klahr and Dunbar, 1-5). Such a theory works and is supported by the data, also it withstands myriad tests (Fiedler and Walther, pp.58). Science is more practical. When a theory works, and keeps working, we consider it right. It serves and works well for all practical purposes. Truth is that which science hopes to asymptotically approach (Orton Anthony, pp.77). Turning back to the main purpose of this research article we emphasize again that the machine and program capacities are limited. They derive from the human capacities which are limited in both directions: towards the extremes of the macro-world that are infinitely great in number and in dimensions; also, towards the extremes of the micro-world that are infinitely great in number and infinitely small in dimensions.

\section{Conclusions}

The computer programs are a very powerful and helpful tool for calculus, plotting, constructing geometric figures, studying the properties of different functions or equations, demonstration in teaching math and other fields of science and so on.

In using $\mathrm{c}^{*}$ omputer programs the teachers must have into consideration that,

1. The potentials of the computer programs are limited

2. The space or the medium in which a computer program operates is discrete or discontinues

3. The students get knowledge regarding the behavior of a function, or the properties of different objects, or calculus, not for every case.

4. The analytical method in calculus gives the right answer, and a mathematically and only a logically shaped mind has the power to pierce the division between points, lines and curves and, discern the elements of the micro-world.

NOTE: Anyone can try him/herself the above examples or others by using GeoGebra software simply by downloading it from www.geogebra.org.

\section{References}

[1] Bruner, J. S., "The act of discovery", Harvard Educational Review, 1961, pp. 21-32.

[2] Fiedler Klaus and Walther Eva, "Stereotyping as Inductive Hypothesis Testing", Psychology Press, Great Britain, 2004, pp. 56-59.

[3] Hulshof C.D., "Discovery of Ideas and Ideas about Discovery", Publisher: Twente University Press, Enschede, the Netherlands (www.tup.utwente.nl), 2001, pp. 16-42.

[4] John Tabak, "Numbers: Computers, Philosophers and the Search for Meaning", The History of Mathematics, Printed in USA, 2004, pp.59, 173.

[5] Josef Böhm, "Linking Geometry, Algebra and Calculus with GeoGebra", ACDCA, DUG and Technical University of Vienna (http://www.geogebra.org), 2008, pp. 3-8.

[6] Klahr, D. and Dunbar, K., "Dual space search during scientific reasoning”, Cognitive Science, 1988, pp. 12, 1-5.

[7] Lu Yu-Wen Allison, "Linking Geometry and Algebra: A multiple-case study of Upper-Secondary mathematics teachers' conceptions and practices of GeoGebra in England and Taiwan", Faculty of Education, University of Cambridge (http://www.geogebra.org), 2008, pp. 1-2, 25-26.

[8] Markus Hohenwartera and Judith Hohenwarter, "Introduction to GeoGebra." (http://www.geogebra.org), 2008, pp. 7-8

[9] Michel Detlefsen, "Proof and Knowledge in Mathematics", Printed in USA and Canada, 1992, Taylor and Francis e-Library, 2005, pp.26.

[10] Orton Anthony, "Learning Mathematics: Issues, Theory and Classroom Practice", British Library Cataloguing-inPublication Data, Third Edition, (www.continuumbooks.com), 2004, pp.71-84.

[11] Reimann P., "Problem solving models of scientific discovery learning processes", Published by P. Lang in Frankfurt am Main, New York, 1991, pp.15-45. 